\title{
Shedding light into the black box of out-of-hospital respiratory distress - a retrospective cohort analysis of discharge diagnoses, prehospital diagnostic accuracy, and predictors of mortality
}

\section{Patrick Spörl ( $\sim$ patrick.spoerl@outlook.de)}

University Hospital RWTH Aachen https://orcid.org/0000-0002-6179-9333

\section{Stefan K. Beckers}

University Hospital RWTH Aachen

Rolf Rossaint

University Hospital RWTH Aachen

Marc Felzen

University Hospital RWTH Aachen

Hanna Schröder

University Hospital RWTH Aachen

Original research

Keywords: Respiratory distress, dyspnea, emergency medical service (EMS), diagnostic accuracy, mortality

Posted Date: February 14th, 2022

DOI: https://doi.org/10.21203/rs.3.rs-956219/v2

License: (c) (i) This work is licensed under a Creative Commons Attribution 4.0 International License. Read Full License

Version of Record: A version of this preprint was published at PLOS ONE on August 3rd, 2022. See the published version at https://doi.org/10.1371/journal.pone.0271982. 


\section{Abstract}

Background: Although respiratory distress is one of the most common complaints of patients requiring emergency medical services (EMS), there is a lack of evidence on important aspects.

Objectives: Our study aims to determine how accurately correct diagnoses are made by EMS physicians in the out-of-hospital setting, identify examination findings that correlate with diagnoses, investigate hospital mortality, and identify mortality-associated predictors.

Methods: This retrospective observational study examined EMS encounters between December 2015 and May 2016 in the city of Aachen, Germany, in which an EMS physician was present at the scene. Adult patients were included if the EMS physician initially detected dyspnea, low oxygen saturation, or pathological auscultation findings at the scene $(n=719)$. The analyses were performed by linking out-ofhospital data to hospital records and using binary logistic regressions.

Results: The overall diagnostic accuracy was $69.9 \%$ (485/694). The highest diagnostic accuracies were observed in asthma $(15 / 15 ; 100 \%)$, hypertensive crisis $(28 / 33 ; 84.4 \%)$, and COPD exacerbation (114/138; $82.6 \%)$, lowest accuracies were observed in pneumonia (70/142; 49.3\%), pulmonary embolism (8/18; $44.4 \%)$, and urinary tract infection $(14 / 35 ; 40 \%)$. The overall hospital mortality rate was $13.8 \%(99 / 719)$. The highest hospital mortality rates were seen in pneumonia $(44 / 142 ; 31 \%)$ and urinary tract infection $(7 / 35 ; 20 \%)$. Identified risk factors for hospital mortality were metabolic acidosis in the initial blood gas analysis (odds ratio (OR) 11.84), the diagnosis of pneumonia (OR 3.22) reduced vigilance (OR 2.58), low oxygen saturation (OR 2.23), and increasing age (OR 1.03 by 1 year increase).

Conclusions: Our data highlight the diagnostic uncertainties and high mortality in out-of-hospital emergency patients presenting with respiratory distress. Pneumonia was the most common and most frequently misdiagnosed cause and showed highest hospital mortality. The identified predictors could help in early detection of patients at risk in the future.

\section{Introduction}

Emergency patients presenting with respiratory distress pose a major challenge to emergency medical service (EMS) professionals. On the one hand, available studies indicate that these patients have a markedly higher mortality rate than patients presenting with other complaints (1-3). On the other hand, the high number of differential diagnoses and limited diagnostic options complicate accurate diagnosis in an out-of-hospital setting. Previous studies conducted in out-of-hospital settings (4-6) and emergency departments $(2,7,8)$ have shown a wide range of possible causes of respiratory distress. This is a relevant issue because the available treatment algorithms are mostly based on the suspected underlying disease. An incorrect suspected diagnosis may result in the initiation of nonindicated procedures and a lack of indicated procedures, leading to a worse outcome (9). Reliable out-of-hospital diagnosis is thus essential for effective out-of-hospital therapy. 
But how accurate are EMS professionals in identifying the correct discharge diagnosis? To date, few studies have drawn conclusions about the diagnostic accuracy of EMS professionals in patients presenting with respiratory distress. Two previous studies highlight that the diagnostic error rates in these emergencies appear to be very high $(10,11)$. However, detailed data on the diagnostic accuracy of specific discharge diagnoses are often missing.

\section{Objectives}

The primary goal of this study is to identify the underlying cause of each analyzed case to determine the diagnostic accuracy of EMS physicians in an out-of-hospital setting. In addition, it should be investigated which initial examination findings correlate with underlying causes. The second focus is to analyze the hospital mortality of this patient population to identify predictors of mortality to detect at-risk patients early in the future.

\section{Materials And Methods}

\section{Study Design}

This retrospective observational study was conducted within the EMS system in the city of Aachen, Germany, during a 6-month observation period (December 2015 to May 2016). All patients who presented with a respiratory problem and were treated by a physician-staffed emergency medical team (PEMT) were included in the analysis. The dataset was collected exclusively for the purpose of this study.

\section{Ethics}

The study plan was submitted to the Ethics Committee of the Medical Faculty of RWTH Aachen University. In a statement dated July 9, 2018, the committee indicated that an application was not necessary and that there were no ethical or legal objections to the study (file number EK 180/18).

\section{EMS system and operating area}

The EMS in Germany is based on a two-tiered care system. An ambulance, manned by two paramedics, is dispatched after receiving an emergency call. The emergency dispatcher can also alert a PEMT staffed by an EMS physician and a paramedic. The PEMT is routinely alerted in potentially life-threatening cases (e.g., suspected cardiac arrest or severe respiratory failure). In addition, it can be requested by an ambulance whenever further medical support is needed at the scene. 
Two 24/7 PEMTs, as well as 16 ambulances, serve the city of Aachen, which has approximately 250,000 inhabitants. All EMS physicians are experienced anesthesiologists, who are in at least the 4th year of their residency at the University Hospital RWTH Aachen. They must have spent at least one year in intensive care medicine and passed an 80-hour certifying course in emergency medicine. Three primary care hospitals and one university hospital were available for admission in the city of Aachen during the study period.

\section{Patient selection and data collection}

We included all PEMT encounters in which patients were at least 18 years old and the EMS physician documented dyspnea, low oxygen saturation (SpO2<90\%), or pathological auscultation findings initially at the scene. Fig 1 lists the inclusion and exclusion criteria we applied to identify adult nontraumatic respiratory emergencies.

Data acquisition was based on all items from the PEMT standardized protocols and was complemented with data from hospital records. The data from hospital records were manually collected and linked in accordance with data protection regulations. For this purpose, all four hospitals in the city of Aachen provided access to their hospital information systems. Data from the following data sources were analyzed: handwritten PEMT standardized protocols, hospital discharge letters, and, if available, digital emergency department protocols as well as initial blood gas analyses.

\section{Terminology used in diagnoses}

Diagnoses suspected by the PEMT are referred to as "out-of-hospital diagnoses" in this paper. The confirmed underlying cause for the emergency call, according to the hospital records, is referred to as "discharge diagnosis". By reviewing the hospital data, we ensured that the diagnoses that resulted from complications during hospitalization were not considered discharge diagnoses. We standardized the terminology of the diagnoses by summarizing synonymous diseases. All discharge diagnoses were classified into one of those shown in Fig 2.

\section{Diagnostic accuracy of the PEMT}

To assess diagnostic accuracy, we analyzed each hospital discharge letter and PEMT protocol and determined the consistency of the diagnoses. We considered each documented out-of-hospital diagnosis and defined it as correct if any of them matched the discharge diagnosis. 
In cases where the PEMT suspected a diagnosis of "non-ST segment elevation acute coronary syndrome" (NSTE-ACS) or "pulmonary embolism", the out-of-hospital diagnosis was defined as correct even if the only discharge diagnosis made was "exclusion of ACS/pulmonary embolism" because exclusion is not possible with certainty in an out-of-hospital setting.

\section{Blood gas analysis (BGA)}

All BGA results were obtained in the emergency departments (to date, blood gas analysis is not available in an out-of-hospital setting in the city of Aachen). It was often unclear whether BGA was performed with arterial, capillary, or venous blood. Therefore, we extended the reference ranges for $\mathrm{pCO} 2$, standard bicarbonate, and lactate to cover blood from all three sources. The reference ranges used are listed in S1 Table. Because the reference ranges for p02 differ widely depending on the source of the blood, we did not consider $\mathrm{pO} 2$.

\section{Statistical analysis}

Statistical analysis was performed using SPSS ${ }^{\circledR}$ version 26 (IBM Corporation, Armonk, New York, USA). Two-sided $p$ values $<0.05$ were considered significant.

\section{Identification of findings upon physical examination associated with discharge diagnoses}

In Fig 4 and S2 Table, we used binary logistic regressions to test out-of-hospital and emergency department examination findings for associations with discharge diagnoses (all evaluated variables are listed below in Fig 4). First, univariable analyses were performed for each diagnosis. Multivariable analysis was performed in the second step to determine which pathological findings were independently associated with their respective discharge diagnoses. For this purpose, all variables with a univariable $p$ value $<0.20$ were considered. Due to the high number of missing data points for some variables (presented in S3 Table), the case count for the multivariable analyses was severely reduced. We considered the resulting risk of incorrect conclusions to be substantial and performed multiple imputations for all examination findings presented in S3 Table to minimize the risk of bias. The missing data were presumed to be missing at random, and 20 imputed datasets were generated using the fully conditional specification method based on the Markov chain Monte Carlo method. Multivariable analyses were performed on all imputed datasets, and the reported data represent the pooled results.

\section{Predictors of hospital mortality}


In Table 2 (listed in detail in S4 Table) we examine the association of predictors with hospital mortality using binary logistic regression. All non-BGA variables (listed in detail below Table 2) were first tested using univariable analysis. In the next step, a multivariable analysis was performed to determine which pathologies were independently associated with hospital mortality. For this purpose, all non-BGA variables with a univariable $p$ value $<0.20$ were included. Because of the many missing data points for examination findings, multiple imputations were performed, analogous to the procedure described above.

To assess the impact of BGA results on hospital mortality, we performed univariable analyses for all BGA results listed in $\mathbf{S 1}$ Table. BGA result with $p<0.2$ in univariable analyses were tested in separate multivariable analyses, which included all non-BGA variables with $p<0.2$ in univariable analysis.

\section{Results}

In total, 928 PEMT encounters were considered according to the inclusion and exclusion criteria. These adult nontraumatic respiratory emergencies represented $24.1 \%$ of all PEMT encounters in the city of Aachen during the study period $(n=3,856)$. The study population consisted of 719 encounters in which hospital records were available.

\section{Characterization of the study population}

Table 1 characterizes the patient population and presents information on PEMT encounters and hospital follow-up.

\section{Table 1. Characterization of patients, PEMT encounters, and hospital follow-up.}




\begin{tabular}{|c|c|}
\hline Age (years), mean (standard deviation) & $70.2(16.7)$ \\
\hline Female sex, n (\%) & $335(46.9)$ \\
\hline \multicolumn{2}{|l|}{ PEMT alerting } \\
\hline Initial alerting on emergency call, $\mathrm{n}(\%)$ & $626(87.1)$ \\
\hline Alerting by ambulance on scene, $\mathrm{n}(\%)$ & $93(12.9)$ \\
\hline \multicolumn{2}{|l|}{ Location of PEMT encounters } \\
\hline Flat, n (\%) & $493(68.6)$ \\
\hline Senior citizen's home, n (\%) & $122(17.0)$ \\
\hline General practice, $\mathrm{n}(\%)$ & $34(4.7)$ \\
\hline Street, $\mathrm{n}(\%)$ & $24(3.3)$ \\
\hline Others, n (\%) & $46(6.4)$ \\
\hline \multicolumn{2}{|l|}{ Frequency of out-of-hospital pathological findings } \\
\hline Dyspnea, n (\%) & $631(87.8)$ \\
\hline Wheezing upon auscultation, n (\%) & $188(26.1)$ \\
\hline Crackles upon auscultation, n (\%) & $181(25.2)$ \\
\hline Peripheral oxygen saturation < 90\%, n (\%) & $308(42.8)$ \\
\hline \multicolumn{2}{|l|}{ Hospital treatment } \\
\hline Outpatient treatment in the emergency department, $\mathrm{n}(\%)$ & $74(10.3)$ \\
\hline Inpatient hospital treatment, $\mathrm{n}(\%)$ & $645(89.7)$ \\
\hline ICU admission, $\mathrm{n}(\%)$ & $161(22.4)$ \\
\hline In-hospital death, n (\%) & $99(13.8)$ \\
\hline Length of stay in hospital (days), mean (standard deviation) & $8.7(12.5)$ \\
\hline
\end{tabular}

PEMT physician-staffed emergency medical team; ICU intensive care unit.

\section{Discharge diagnoses and diagnostic accuracy of the PEMT}

A total of 793 discharge diagnoses were found. Hospital records defined two discharge diagnoses in 92 cases, three in one case, and the discharge diagnosis remained unclear in 19 cases. Pneumonia $(n=142$; $17.9 \%)$, COPD exacerbation ( $n=138 ; 17.4 \%)$, and decompensated heart failure $(n=125 ; 15.8 \%)$ accounted for $51.1 \%(n=405)$ of all discharge diagnoses. Fig 2 lists all discharge diagnoses made in our 
study and shows the diagnostic accuracy of the PEMT. Further descriptive data on demographics, physical examination findings, and hospitalization details for the most frequent discharge diagnoses are presented in S5 Table.

Overall, the diagnostic agreement between out-of-hospital diagnoses and discharge diagnoses was $69.9 \%(n=485)$. Diagnostic accuracy could not be assessed in 25 of 719 encounters (3.5\%) because either no out-of-hospital diagnosis was documented $(n=6)$ or the discharge diagnosis remained unclear $(n=19)$. Therefore, diagnostic accuracy analysis was performed on the remaining 694 encounters.

Fig 3 shows which out-of-hospital diagnoses the PEMTs suspected when they failed to identify the correct discharge diagnosis.

\section{Characterization of discharge diagnoses and identification of associated findings upon physical examination}

Fig 4 displays all out-of-hospital and emergency department examination findings that showed an independent association with the diagnoses evaluated. Detailed results of the statistical analysis are provided in $\mathbf{S} 2$ Table.

We found that some initial out-of-hospital examination findings were not documented (and probably not measured) in a relevant proportion of PEMT encounters (S3 Table). For example, respiratory rate was not documented in $26.1 \%$, body temperature in $35.3 \%$, and numeric rating scale in $39.9 \%$ of encounters.

\section{Hospital mortality and associated predictors}

The overall hospital mortality rate in our study was 13.8\% (99/719). Fig 5 shows the hospital mortality rates of the most frequent discharge diagnoses and in how many of the lethal outcomes the PEMT made an incorrect out-of-hospital diagnosis.

In Table 2, we reviewed predictors for their association with hospital mortality. In multivariable analysis, reduced vigilance (GCS < 15), low oxygen saturation (SpO2<90\%), and increasing age were associated with a higher risk of death, whereas wheezing upon auscultation was associated with lower mortality. The association between age and mortality was particularly evident for the 80 - to 89 -year-old group, while 
it was not seen for those over 89 years old (for further details see S4 Table). Moreover, the discharge diagnoses of pneumonia was associated with a significantly higher risk of hospital death.

Meanwhile, an incorrect out-of-hospital diagnosis by the PEMT showed no significant effect on hospital mortality in multivariable analysis.

\section{Hospital mortality and initial blood gas analysis}

Initial blood gas analyses were not performed in half of the cases. $\mathrm{PH}$ and $\mathrm{pCO} 2$ results were available in 364 of 719 cases $(50.6 \%)$, and standard bicarbonate was available in 342 cases (47.6\%). Because lactate measurement was exclusively measured at the participating university hospital, lactate results were available only in 192 cases (26.7\%).

Analysis of the association between initial BGA and hospital mortality showed that metabolic acidosis significantly increased the risk of death. (Table 2). This association was evident in all BGA results related to metabolic acidosis (decreased $\mathrm{pH}$, decreased standard bicarbonate, and increased lactate). More detailed results are shown in $\mathbf{S 1}$ Table.

\section{Table 2. Predictors of hospital mortality.}




\begin{tabular}{|c|c|c|}
\hline & OR $(95 \% \mathrm{CI})$ & $\mathrm{p}$ value \\
\hline \multicolumn{3}{|l|}{ Age } \\
\hline Age by 1 year increase & $1.031(1.007-1.055)$ & 0.011 \\
\hline Age 80-89 years & $1.868(1.033-3.378)$ & 0.039 \\
\hline \multicolumn{3}{|l|}{ Pathological examination findings } \\
\hline Reduced vigilance $(\mathrm{GCS}<15)$ & $2.583(1.341-4.976)$ & 0.005 \\
\hline Low oxygen saturation (SpO2 < 90\%) & $2.231(1.193-4.173)$ & 0.012 \\
\hline Wheezing upon auscultation in the emergency department & $0.362(0.136-0.961)$ & 0.041 \\
\hline \multicolumn{3}{|l|}{ Discharge diagnoses } \\
\hline Pneumonia & $3.221(1.562-6.644)$ & 0.002 \\
\hline \multicolumn{3}{|l|}{ BGA results } \\
\hline $\mathrm{pH}<7.35$ & $4.494(1.740-11.608)$ & 0.002 \\
\hline $\mathrm{pH}<7.30$ & $3.751(1.346-10.457)$ & 0.012 \\
\hline Standard bicarbonate by $1 \mathrm{mmol} / \mathrm{L}$ increase & $0.903(0.827-0.986)$ & 0.023 \\
\hline Standard bicarbonate $<17 \mathrm{mmol} / \mathrm{L}$ & $13.124(1.970-87,415)$ & 0.008 \\
\hline Lactate by $1 \mathrm{mmol} / \mathrm{L}$ increase & $1.506(1.072-2.117)$ & 0.018 \\
\hline Lactate $>4.0 \mathrm{mmol} / \mathrm{L}$ & $5.694(1.066-30.423)$ & 0.042 \\
\hline Metabolic acidosis* & $11.841(2.195-63.881)$ & 0.004 \\
\hline
\end{tabular}

Binary logistic regression - results of multivariable analysis

First, univariable analyses were performed evaluating the following potential risk factors for hospital mortality: age, sex, discharge diagnoses with $n>10$, out-of-hospital misdiagnosis by the PEMT, out-of-hospital findings: systolic blood pressure $<100 \mathrm{mmHg}$, heart rate $>100 / \mathrm{min}$, peripheral oxygen saturation $<90 \%$, respiratory rate $\geq 22 / \mathrm{min}$, body temperature $\geq 38{ }^{\circ} \mathrm{C}$, body temperature $\leq 36{ }^{\circ} \mathrm{C}$, Glasgow Coma Scale $<15$, numeric rating scale $\geq 1$, crackles upon auscultation, wheezing upon auscultation, emergency department findings: crackles upon auscultation, wheezing upon auscultation, silent lung upon auscultation, lower extremity edema, and BGA results (listed in S1 Table).

Second, a multivariable analysis was performed including all non-BGA variables with $p<0.2$ in univariable analyses.

All BGA result with $p<0.2$ in univariable analyses were tested in separate multivariable analyses including all non$B G A$ variables with $p<0.2$ in the univariable analysis. Table 2 lists all significant results in multivariable analyses ( $p<$ 0.05). Detailed results of logistic regressions are presented in S1 and S4 Tables.

OR: odds ratio; 95\% CI: 95\% confidence interval of OR; GCS: Glasgow Coma Scale; SpO2: peripheral oxygen saturation; pH: hydrogen potential; pCO2: partial pressure of carbon dioxide; HCO3: standard bicarbonate; OR: odds 
ratio; 95\% CI: 95\% confidence interval of OR; * Metabolic acidosis is defined as: $p H<7.35$ and HCO3 $<21 \mathrm{mmol} / \mathrm{L}$ and pCO2 $\leq 6.7 \mathrm{kPa}(50 \mathrm{mmHg})$ or $\mathrm{pH}<7.35$ and $\mathrm{pCO} 2 \leq 6.7 \mathrm{kPa}(50 \mathrm{mmHg})$ and lactate $>5.0 \mathrm{mmol} / \mathrm{L}$.

\section{Discussion}

This study of patients presenting with respiratory distress examined discharge diagnoses, the diagnostic accuracy of EMS physicians in an out-of-hospital setting, and hospital mortality. Our data show that diagnostic accuracy and hospital mortality differ widely depending on the discharge diagnoses. Overall, the high portions of misdiagnosis and the high hospital mortality rate confirm the assumption that this patient population seems to be particularly challenging and critically ill.

\section{Discharge diagnoses and diagnostic accuracy of the PEMT}

The high proportion of $24.1 \%$ of all PEMT encounters during the study period shows the great relevance of nontraumatic respiratory emergencies in the daily routine of EMS. The highest prevalence was found for pneumonia, COPD exacerbation, and decompensated heart failure. This is largely consistent with the results of comparable out-of-hospital $(5,6)$ and emergency department $(7,8)$ studies. Seven other discharge diagnoses accounted for more than $25 \%$ of PEMT encounters in our study. This demonstrates that EMS professionals must consider a wide range of differential diagnoses when treating patients with respiratory distress.

Studies assessing the diagnostic accuracy of EMS professionals in respiratory emergencies are rare and differ in the way diagnostic accuracy was calculated.

In our study, the overall proportion of PEMT misdiagnoses was $30.1 \%$. In two former PEMT studies from Germany, the highest portions for misdiagnosis ( $26 \%$ and $41 \%$ ) were found in patients admitted for dyspnea $(10,11)$. In contrast, two other German PEMT studies observed the highest misdiagnosis rates in neurological emergencies $(12,13)$. Interestingly, these studies did not show a notable accumulation of misdiagnoses in respiratory emergencies. It is noticeable that one of them - the study by Arntz et al. (12) - differs in the qualifications of the EMS physicians involved. While $68 \%$ of the EMS physicians in this study were internists, only anesthesiologists worked as EMS physicians in all other studies (including ours). The strikingly low misdiagnosis rate described by Arntz et al. may be because internist-trained EMS physicians have more clinical experience in diagnosing patients with respiratory problems.

Looking at the individual discharge diagnoses in our study, there were huge differences in diagnostic confidence. Low diagnostic accuracy is particularly notable for pneumonia, which was the most frequent discharge diagnosis in our study. Pneumonia was also associated with the highest hospital mortality rate 
(31\%), the highest rate of ICU admissions (37.3\%), and a significantly increased likelihood of inhospital death. This observation is supported by the paramedic study by Kauppi et al., which also found that patients with respiratory distress due to pneumonia had the highest mortality (5). Our analysis of PEMT misdiagnoses found that EMS physicians often suspected COPD exacerbation or decompensated heart failure when pneumonia was actually present.

It is less surprising that diagnostic accuracy was low for urinary tract infection. Presumably, few EMS professionals think primarily of a urinary tract infection in patients presenting with respiratory distress. However, patients admitted with urinary tract infection showed the second highest mortality rate of $20 \%$. Closer examination of these cases revealed that a second discharge diagnosis (e.g., COPD exacerbation, pneumonia, and decompensated heart failure) was made in 12 of 35 patients admitted with a urinary tract infection. In these cases, it cannot be excluded that the respiratory symptoms were caused by these second discharge diagnoses. Urinary tract infections were at least concomitantly present in $4.9 \%$ of analyzed PEMT encounters and should therefore be considered a relevant differential diagnosis and cause for respiratory distress.

One former emergency department study of elderly patients with respiratory distress described that misdiagnosis resulted in worse patient outcomes (9). In our study, the EMS physician made an incorrect diagnosis in $41.4 \%$ of all cases of in-hospital death. The significant association between out-of-hospital misdiagnosis and hospital mortality in the univariable analysis, was not demonstrated in the multivariable analysis. Nevertheless, it seems plausible that an increase in diagnostic accuracy would result in a decrease in morbidity and mortality.

\section{Pathological findings associated with discharge diagnoses}

The two strongest associations we found were of prehospital wheezing being strongly associated with asthma (odds ratio 38.8) and lower extremity edema being associated with decompensated heart failure (odds ratio 13.7). Our data indicate that a clear recommendation can be made for thorough auscultation of the lungs and evaluation of lower extremity edema in all patients with respiratory distress.

Several findings correlated with low or moderate odds ratios with discharge diagnoses. It became clear that many routine parameters (e.g., body temperature and GCS) provide important information about underlying diagnoses. Nevertheless, some of these parameters were not documented in many encounters. 
Our data indicate that a thorough and complete physical examination, as well as consideration of the numerous differential diagnoses are requirements for making a reliable out-of-hospital diagnosis, especially in cases of respiratory distress. This even allows for the identification of unexpected pathological findings (e.g., high body temperature), which can provide essential hints for the correct diagnosis.

In our opinion, the prehospital established ABCDE approach (airway, breathing, circulation, disability, environment) is suitable for making reliable diagnoses even in patients with respiratory distress, as long as followed conscientiously. In this regard, EMS professionals should be sensitized that a thorough search, especially for B and C problems, is essential for a correct diagnosis. Moreover, focused education and training could help EMS professionals be better prepared to handle these difficult emergencies in the future.

Several emergency department studies have shown that the use of point-of-care ultrasound (POCUS) in respiratory emergencies can reduce the number of differential diagnoses and increase diagnostic accuracy (14-16). Out-of-hospital use of ultrasound and training of EMS professionals in POCUS therefore have the potential to increase diagnostic confidence.

\section{Hospital mortality and the search for associated predictors}

The hospital mortality rate in our study was $13.8 \%$, which was comparable to that of three similar EMS studies from Scandinavia involving patients with dyspnea $(1,4,5)$. These studies reported hospital or 30day mortality rates of $11 \%, 12.3 \%$ and $13.2 \%$, respectively. We attribute the slightly higher mortality in our study to the fact that only EMS encounters involving EMS physicians were analyzed and that these encounters presumably represent a particularly critical subset of all respiratory emergencies. Unsurprisingly, the ICU admission rate in our study was also very high (22.4\%). The consistent aboveaverage mortality and ICU admission rates highlight that patients with respiratory distress should be considered high-risk.

According to our analysis, decreased vigilance, low oxygen saturation, and increasing age can be considered independent predictors for hospital mortality. Two former emergency department studies that included patients with any complaint also found that decreased vigilance was an independent risk factor for 30-day mortality $(3,17)$. The same conclusion was made in an EMS study of patients with the complaint of dyspnea $(18)$. Two of these studies $(3,18)$ similarly reported that low oxygen saturation was 
a risk factor for mortality. Patients with dyspnea have a higher risk of death with increasing age, which has also been shown by previous studies $(9,18)$.

\section{Hospital mortality and initial blood gas analysis}

Examination of the initial BGA results shows that metabolic acidosis appears to be a strong risk factor for death.

Surprisingly, there is little evidence of the prognostic value of BGA results in patients with respiratory distress. Two previous studies showed that acidosis led to a higher risk of mortality or ICU admission in patients with dyspnea $(19,20)$. That initial hyperlactatemia is an independent predictor of mortality in unselected emergency department patients has been demonstrated by numerous studies (21-23). Our results highlight that elevated lactate is an important prognostic parameter even in emergency patients with respiratory distress. Therefore, BGA, including lactate, should be routinely measured in all patients presenting with respiratory distress.

\section{Limitations}

Compared to other studies, our study is based on a relatively small sample size. Because we examined only PEMT encounters during winter and spring, our results do not allow for conclusions about all respiratory emergencies throughout an entire year. Rather, it can be assumed that we studied a particularly critically ill subset of this patient population. Since the evaluation of diagnostic accuracy was not blinded, it cannot be excluded with certainty that it was over- or underestimated. The many missing data in some examination findings posed challenges for the multivariable analyses. We tried to compensate them by running multiple imputations. The sample size for BGA analysis was particularly small, as BGA results were obtained from only half and lactate was measured in a quarter of the encounters.

\section{Conclusion}

We showed that the overall diagnostic uncertainty in patients with respiratory distress was high but varied greatly between discharge diagnoses. Misdiagnoses occurred particularly frequently in patients with pneumonia. At the same time, pneumonia was responsible for most EMS encounters, most ICU admissions, and most hospital deaths.

Analysis of hospital mortality shows that emergency patients presenting with respiratory distress have a markedly high risk of in-hospital death. According to our data, decreased vigilance, low oxygen saturation, increasing age, and metabolic acidosis can be considered risk factors for hospital mortality. 
Study findings call for a thorough physical examination and consideration of the many differential diagnoses to increase diagnostic accuracy in the future.

\section{Declarations}

\section{Acknowledgments}

The authors would like to thank the representatives of the participating hospitals for their kind cooperation in evaluating the hospital records. In particular, we would like to thank Mrs. Ulrike Brocker, Mrs. Monique Tasidis, and Dr. Michael Schöntag for their capable and patient support. Furthermore, we thank Dr. Martin Klasen for his competent support in planning the statistical analysis.

\section{Declaration of interests}

none.

\section{Data availability statement}

The datasets used and analyzed during the current study are available from the corresponding author upon reasonable request.

\section{Funding}

This research did not receive any specific grant from funding agencies in the public, commercial, or notfor-profit sectors.

\section{Authors' contributions}

PS, SKB, HS, and MF planned the study design. PS collected the data, performed the statistical analysis, and interpreted the results. PS, MF, SKB, HS and RR discussed the findings and their interpretation. PS wrote the draft of the manuscript. MF, SKB, HS, and RR contributed to the correction of the manuscript. All authors approved the submitted version and all modified versions of the manuscript.

\section{References}

1. Bøtker MT, Terkelsen CJ, Sørensen JN, Jepsen SB, Johnsen SP, Christensen EF, et al. Long-Term Mortality of Emergency Medical Services Patients. Ann Emerg Med. 2017;70(3):366-73.e3. 
2. Mockel M, Searle J, Muller R, Slagman A, Storchmann H, Oestereich P, et al. Chief complaints in medical emergencies: do they relate to underlying disease and outcome? The Charité Emergency Medicine Study (CHARITEM). Eur J Emerg Med. 2013;20(2):103-8.

3. Barfod C, Lauritzen MM, Danker JK, Sölétormos G, Forberg JL, Berlac PA, et al. Abnormal vital signs are strong predictors for intensive care unit admission and in-hospital mortality in adults triaged in the emergency department - a prospective cohort study. Scand J Trauma Resusc Emerg Med. 2012;20:28.

4. Lindskou TA, Pilgaard L, Søvsø MB, Kløjgård TA, Larsen TM, Jensen FB, et al. Symptom, diagnosis and mortality among respiratory emergency medical service patients. PLoS One. 2019;14(2):e0213145.

5. Kauppi W, Herlitz J, Magnusson C, Palmér L, Axelsson C. Characteristics and outcomes of patients with dyspnoea as the main symptom, assessed by prehospital emergency nurses- a retrospective observational study. BMC Emerg Med. 2020;20(1):67.

6. Prekker ME, Feemster LC, Hough CL, Carlbom D, Crothers K, Au DH, et al. The epidemiology and outcome of prehospital respiratory distress. Acad Emerg Med. 2014;21(5):543-50.

7. Kelly AM, Holdgate A, Keijzers G, Klim S, Graham CA, Craig S, et al. Epidemiology, prehospital care and outcomes of patients arriving by ambulance with dyspnoea: an observational study. Scand J Trauma Resusc Emerg Med. 2016;24(1):113.

8. Laribi S, Keijzers G, van Meer O, Klim S, Motiejunaite J, Kuan WS, et al. Epidemiology of patients presenting with dyspnea to emergency departments in Europe and the Asia-Pacific region. Eur $\mathrm{J}$ Emerg Med. 2019;26(5):345-9.

9. Ray P, Birolleau S, Lefort $Y$, Becquemin $\mathrm{MH}$, Beigelman $\mathrm{C}$, Isnard R, et al. Acute respiratory failure in the elderly: etiology, emergency diagnosis and prognosis. Crit Care. 2006;10(3):R82.

10. Schewe JC, Kappler J, Dovermann K, Graeff I, Ehrentraut SF, Heister U, et al. Diagnostic accuracy of physician-staffed emergency medical teams: a retrospective observational cohort study of prehospital versus hospital diagnosis in a 10-year interval. Scand J Trauma Resusc Emerg Med. 2019;27(1):36.

11. Peter J. Qualität notärtzlicher Diagnosen: Ein Vergleich von Fachärzten und Weiterbildungsassistenten der Anästhesie. Medical doctoral thesis. Friedrich-Alexander-Universität Erlangen-Nürnberg. Germany. 2009. urn:nbn:de:bvb:29-opus-17286. Available from: https://opus4.kobv.de/opus4-fau/frontdoor/index/index/docld/1170

12. Arntz HR, Klatt S, Stern R, Willich SN, Beneker J. Are emergency physicians' diagnoses accurate? Anaesthesist. 1996;45(2):163-70.

13. Heuer JF, Gruschka D, Crozier TA, Bleckmann A, Plock E, Moerer O, et al. Accuracy of prehospital diagnoses by emergency physicians: comparison with discharge diagnosis. Eur J Emerg Med. 2012;19(5):292-6. 
14. Laursen CB, Sloth E, Lassen AT, Christensen R, Lambrechtsen J, Madsen PH, et al. Point-of-care ultrasonography in patients admitted with respiratory symptoms: a single-blind, randomised controlled trial. Lancet Respir Med. 2014;2(8):638-46.

15. Ahn JH, Jeon J, Toh HC, Noble VE, Kim JS, Kim YS, et al. SEARCH 8Es: A novel point of care ultrasound protocol for patients with chest pain, dyspnea or symptomatic hypotension in the emergency department. PLoS One. 2017;12(3):e0174581.

16. Buhumaid RE, St-Cyr Bourque J, Shokoohi H, Ma IWY, Longacre M, Liteplo AS. Integrating point-ofcare ultrasound in the ED evaluation of patients presenting with chest pain and shortness of breath. Am J Emerg Med. 2019;37(2):298-303.

17. Hong W, Earnest A, Sultana P, Koh Z, Shahidah N, Ong ME. How accurate are vital signs in predicting clinical outcomes in critically ill emergency department patients. Eur J Emerg Med. 2013;20(1):27-32.

18. Kauppi W, Herlitz J, Karlsson T, Magnusson C, Palmér L, Axelsson C. Pre-hospital predictors of an adverse outcome among patients with dyspnoea as the main symptom assessed by pre-hospital emergency nurses - a retrospective observational study. BMC Emerg Med. 2020;20(1):89.

19. Burri E, Potocki M, Drexler B, Schuetz P, Mebazaa A, Ahlfeld U, et al. Value of arterial blood gas analysis in patients with acute dyspnea: an observational study. Crit Care. 2011;15(3):R145.

20. Gourhant V, Vuillot O, Claret PG, Lefebvre S, Schaub R, Flacher A, et al. Arterial pH selectively predicts critical care needs in emergency department obese patients with acute dyspnea: A prospective comparative study. Am J Emerg Med. 2019;37(1):67-72.

21. Bernhard M, Döll S, Kramer A, Weidhase L, Hartwig T, Petros S, et al. Elevated admission lactate levels in the emergency department are associated with increased 30-day mortality in non-trauma critically ill patients. Scand J Trauma Resusc Emerg Med. 2020;28(1):82.

22. Barfod C, Lundstrøm LH, Lauritzen MM, Danker JK, Sölétormos G, Forberg JL, et al. Peripheral venous lactate at admission is associated with in-hospital mortality, a prospective cohort study. Acta Anaesthesiol Scand. 2015;59(4):514-23.

23. Bou Chebl R, El Khuri C, Shami A, Rajha E, Faris N, Bachir R, et al. Serum lactate is an independent predictor of hospital mortality in critically ill patients in the emergency department: a retrospective study. Scand J Trauma Resusc Emerg Med. 2017;25(1):69.

\section{Abbreviations}

ACS: acute coronary syndrome;

BGA: blood gas analysis; 
Cl: confidence interval;

COPD: chronic obstructive pulmonary disease;

EMS: emergency medical service;

GCS: Glasgow Coma Scale;

HCO3: standard bicarbonate;

ICU: intensive care unit;

NRS: numeric rating scale;

NSTE-ACS: non-ST segment elevation acute coronary syndrome;

OR: odds ratio;

pC02: partial pressure of carbon dioxide;

pH: hydrogen potential;

POCUS: point-of-care ultrasound;

PEMT: physician-staffed emergency medical team;

p02: partial pressure of oxygen;

Sp02: peripheral oxygen saturation;

STEMI: ST-elevation myocardial infarction

\section{Figures}




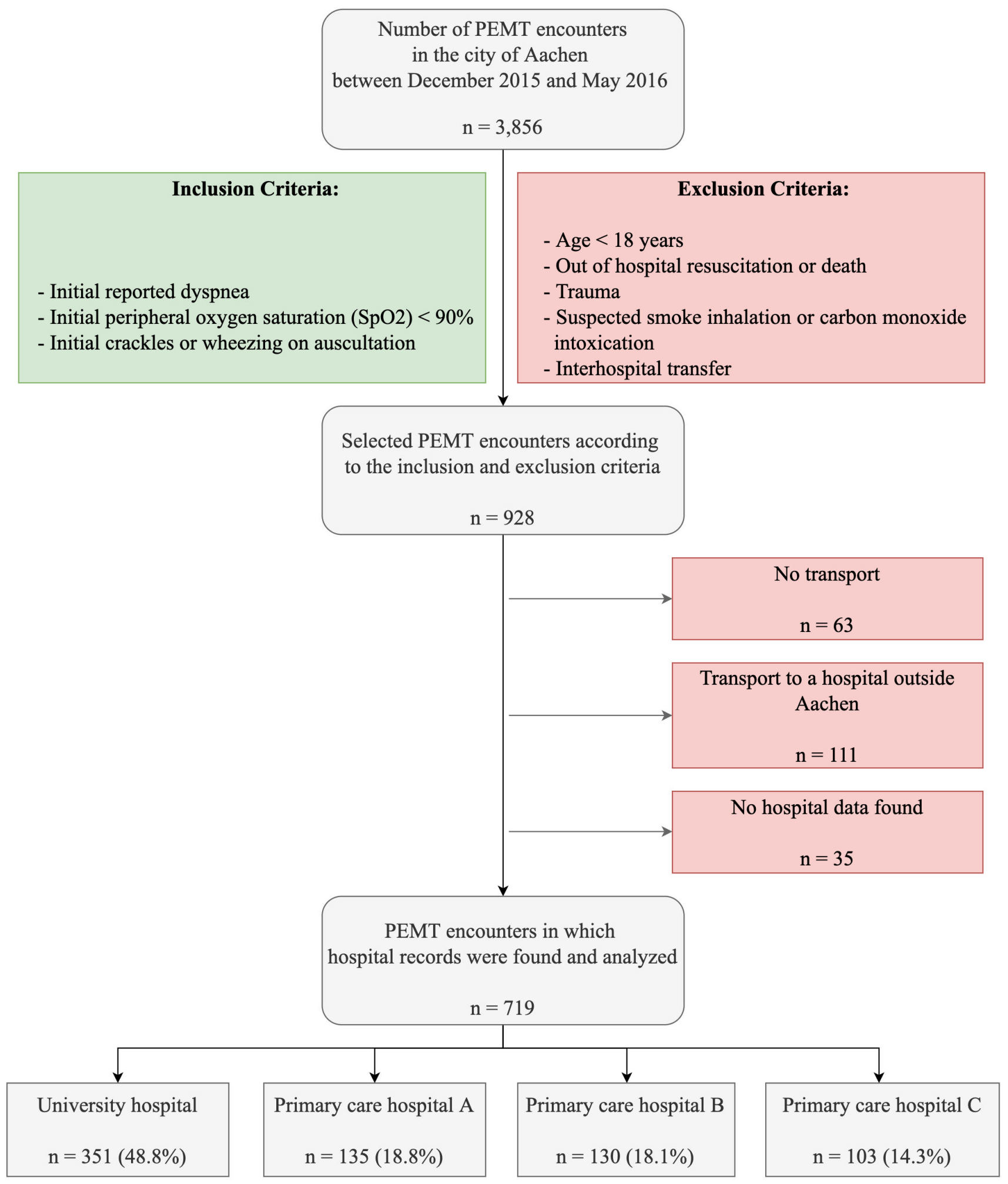

\section{Figure 1}

Study population considering the inclusion and exclusion criteria.

PEMT physician-staffed emergency medical team. 
Diagnostic accuracy not displayed

Incorrect out-of-hospital diagnosis by the PEMT

Correct out-of-hospital diagnosis by the PEMT

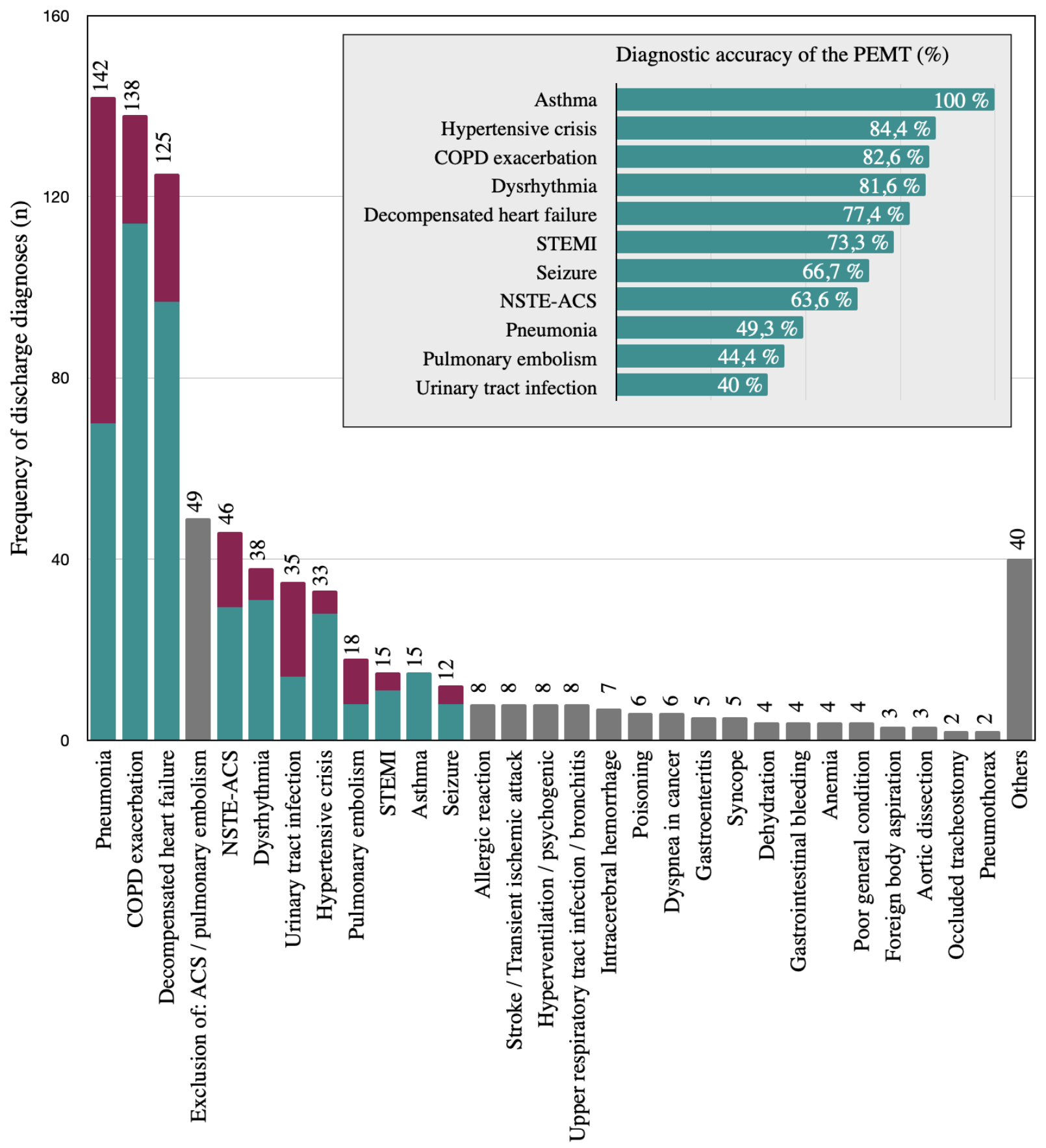

Figure 2

Frequency of discharge diagnoses and diagnostic accuracy of the PEMT.

PEMT: physician-staffed emergency medical team; COPD: chronic obstructive pulmonary disease; ACS: acute coronary syndrome; NSTE-ACS: non-ST segment elevation ACS; STEMI: ST-elevation myocardial infarction; total of number of discharge diagnoses made: 793. 


\section{Pneumonia}

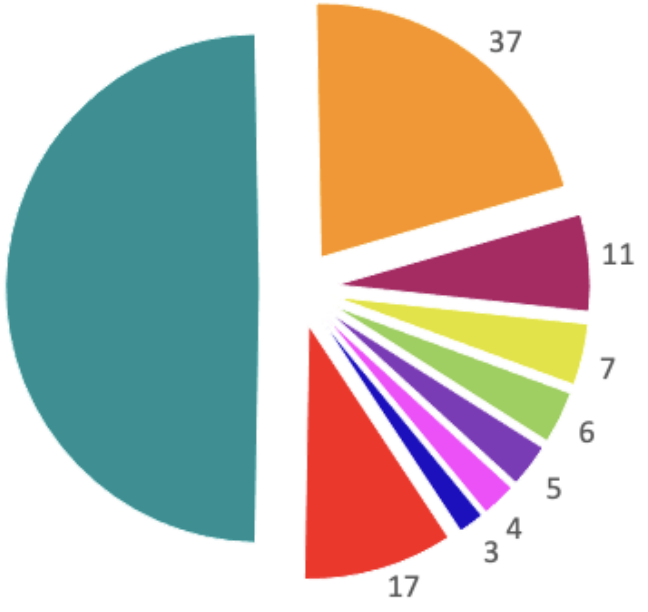

Decompensated heart failure

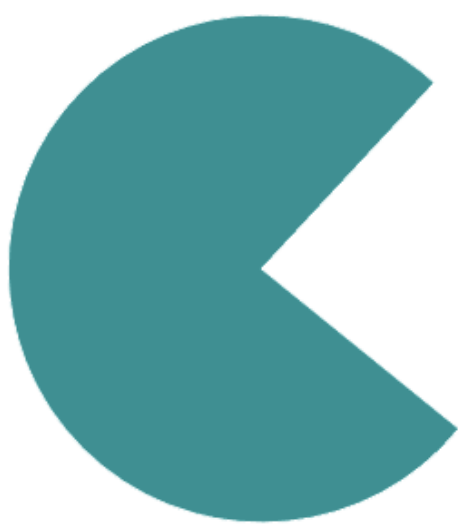

COPD exacerbation

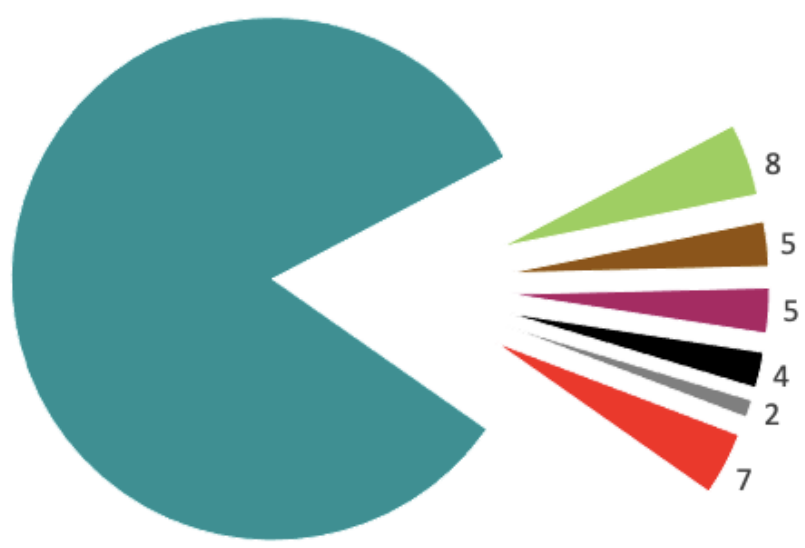

Correct out-of-hospital diagnosis by the PEMT

Incorrect out-of-hospital diagnosis by the PEMT (n): COPD exacerbation

Decompensated heart failure

Upper respiratory tract infection / bronchitis

Acute coronary syndrome

Dysrhythmia

Dulmonary embolism

-Poor general condition

Asthma

-Hypertensive crisis

aPneumonia

DOthers

\section{Figure 3}

\section{Which out-of-hospital diagnoses did the PEMTs suspect in cases of misdiagnosis?}

Shown are the PEMTs' incorrect suspected out-of-hospital diagnoses for the three most frequent discharge diagnoses; PEMT: physician-staffed emergency medical team; COPD: chronic obstructive pulmonary disease. 

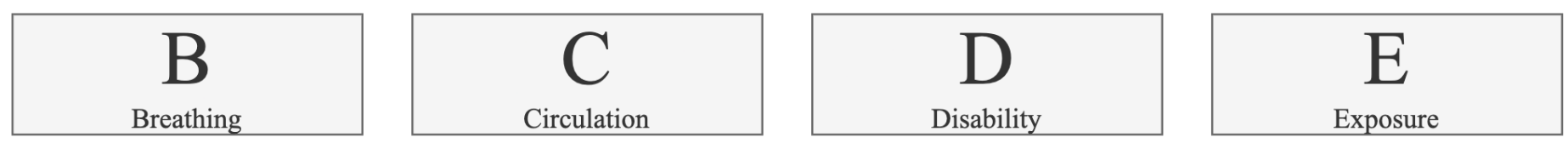

\section{Auscultation findings?}

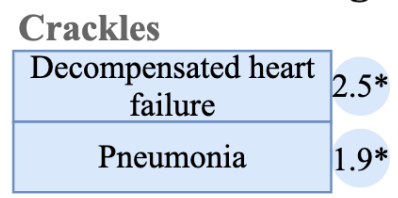

Wheezing

\begin{tabular}{|c|c|}
\hline Asthma & 38.8 \\
\cline { 1 - 1 } COPD exacerbation & $3.9^{*}$ \\
\cline { 1 - 1 } $\begin{array}{c}\text { Decompensated heart } \\
\text { failure }\end{array}$ & $0.4^{*}$ \\
\hline
\end{tabular}

Silent lung

COPD exacerbation 4.0

\section{Low oxygen saturation?}

\section{Hypotension?}

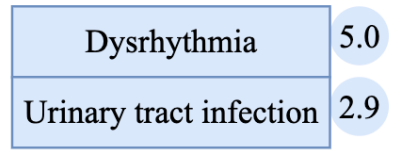

\section{Tachycardia?}

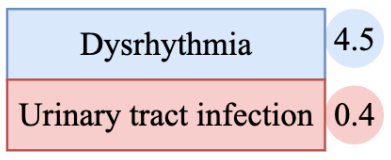

\section{Lower extremity edema?}

\begin{tabular}{|c|c|}
$\begin{array}{c}\text { Decompensated heart } \\
\text { failure }\end{array}$ & 13.7 \\
\cline { 1 - 1 } Dysrhythmia & 3.8 \\
\hline
\end{tabular}

\section{Reduced vigilance?}

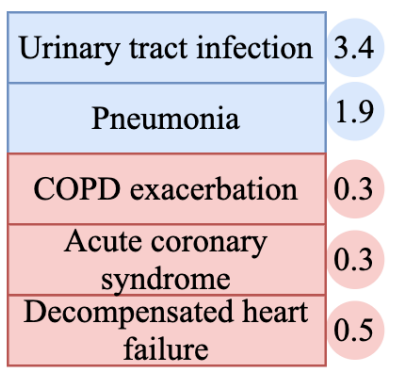

\section{High temperature?}

\begin{tabular}{ll} 
Urinary tract infection 2.3 \\
\hline
\end{tabular}

\section{Reported pain?}

\begin{tabular}{|c|}
\hline $\begin{array}{c}\text { Acute coronary } \\
\text { syndrome }\end{array}$ \\
\hline
\end{tabular}

\begin{tabular}{|c|c|}
\hline Pneumonia & 2.0 \\
\cline { 1 - 1 } Dysrhythmia & 0.3 \\
\cline { 1 - 1 } &
\end{tabular}

\section{Tachypnea?}

Dysrhythmia

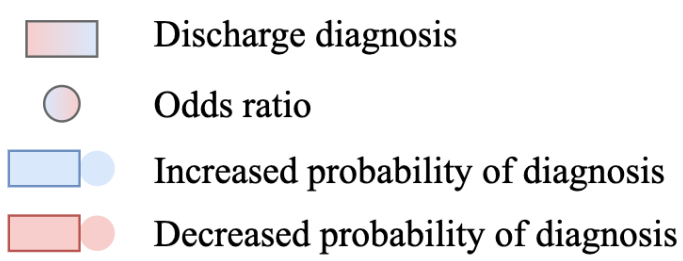

\section{Figure 4}

\section{Associations of initial examination findings and discharge diagnoses.}

Binary logistic regression; only significant results are displayed * mean of the odds ratios (when identical auscultation findings in the out-of-hospital setting and in the emergency department yielded significant results). The following variables were reviewed for associations with discharge diagnoses: out-of-hospital findings: hypotension (systolic blood pressure $<100 \mathrm{mmHg}$ ), tachycardia (heart rate $>100 / \mathrm{min}$ ), low oxygen saturation (peripheral oxygen saturation $<90 \%$ ), tachypnea (respiratory rate $\geq 22 / \mathrm{min}$ ), high temperature (body temperature $\geq 38^{\circ} \mathrm{C}$ ), body temperature $\leq 36^{\circ} \mathrm{C}$, reduced vigilance (Glasgow Coma Scale $<15$ ), reported pain (numeric rating scale $\geq 1$ ), crackles upon auscultation, wheezing upon auscultation, emergency department findings: crackles upon auscultation, wheezing upon auscultation, silent lung upon auscultation, and lower extremity edema. Detailed results of logistic regressions are shown in S2 Table. 


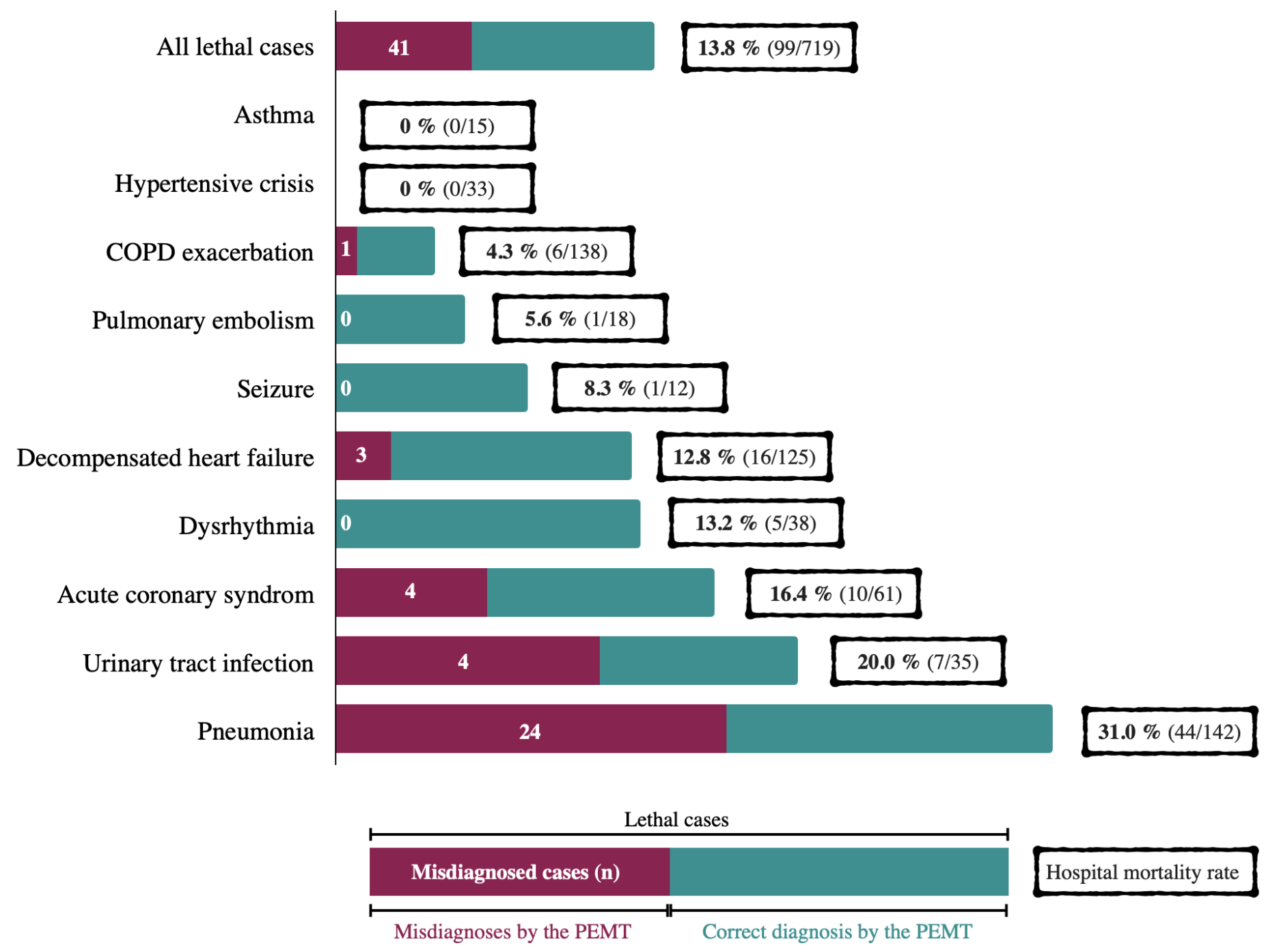

\section{Figure 5}

\section{Hospital mortality and misdiagnoses by the PEMT.}

Shown are the hospital mortality rates for the most frequent discharge diagnoses and for all cases as well as the number of out-of-hospital misdiagnosed cases in lethal outcomes.

PEMT: physician-staffed emergency medical team; COPD: chronic obstructive pulmonary disease.

\section{Supplementary Files}

This is a list of supplementary files associated with this preprint. Click to download.

- Supportinginformation.docx 